\title{
Kinetics of the Addition Reactions of Thiobenzoic Acids to Styrenes or Ethynylbenzenes as the Model of Polyaddition. Study on the Rate-Determining Step and Substituent Effect
}

\author{
Eiichi Kobayashi, * Masaki Sakakibara, Takatsugu Obata, \\ Sadahito Aoshima, and Junji Furukawa \\ Department of Industrial Chemistry, Faculty of Science and Technology, \\ Science University of Tokyo, Noda, Chiba 278, Japan
}

(Received October 27, 1992)

\begin{abstract}
The reaction mechanism and substituent effect on the addition reaction of thiobenzoic acid to styrene or ethynylbenzene were investigated in detail. Both addition reactions proceeded by a radical chain mechanism, and the rate-determining step was the chain transfer step between each intermediate carbon radical $\left[\phi \dot{\mathrm{C}} \mathrm{HCH}_{2} \mathrm{SC}(=\mathrm{O}) \phi\right.$ or $\left.\phi \dot{\mathrm{C}}=\mathrm{CHSC}(=\mathrm{O}) \phi\right]$ and thiobenzoic acid. The substituent effect on the chain transfer step between the para-X substituted intermediate carbon radicals and para-Y thiobenzoic acids in the styrene and ethynylbenzene systems was better correlated by the modified Hammett's equation considering resonance effect than by the simple Hammett's equation. In both styrene and ethynylbenzene systems, the reaction constant $\rho$ for $p$-X intermediate carbon radicals was negative, and that for $p$-Y thiobenzoic acids was positive, suggesting that a perturbation between SOMO of each intermediate carbon radical and LUMO of thiobenzoic acid in the transition state is the important controlling factor for reactivity in the adduct formation of thiobenzoic acid with styrene or ethynylbenzene. That is, the intermediate carbon radicals are nucleophilic. Therefore, the overall reaction rate of the adduct formation increases by introducing a strong electron-donating $(+I)$ group into the para position of styrene or ethynylbenzene and a large $-I$ group into the para position of thiobenzoic acid. The results are compared with those of the addition reaction of thiophenol to styrene or ethynylbenzene. The polyaddition mechanism of 1,4-benzenebiscarbothioic acid to 1,4-divinylbenzene or 1,4-diethynylbenzene is discussed.

KEY WORDS Kinetics / Polyaddition / Addition Reaction / Reaction Mechanism / Substituent Effect / Hammett Correlation / Thiobenzoic Acids / Styrenes / Ethynylbenzenes / Thiophenols /
\end{abstract}

The radical addition reactions of thiols to olefins or acetylenes are well known and used widely to prepare various sulfide compounds and polysulfides. Similar addition reactions have been reported with carbothioic acid (thiol acid) compounds. ${ }^{1}$ For example, Koening et $a .^{2}$ synthesized thioesters by the radical addition reaction of thioacetic acid to vinyl compounds. The analogous reaction with acetylenes gave the $1: 1$ and $2: 1$ adducts with thioacetic acid and acetylenes. ${ }^{3}$ In a similar manner, Marvel et al. ${ }^{4}$ reported polythioesters prepared by the radical polyaddition of biscarbothioic acids to non-conjugated diolefins, and the obtained polymers had higher melting points than the oxygen-containing analogous polymers.

Recently, the authors investigated the polyaddition of 1,4-benzenebiscarbothioic acid (BDTC) to 1,4-divinylbenzene (DVB) ${ }^{5}$ or $1,4-$ diethynylbenzene (DEB). ${ }^{6}$ The polyaddition took place successfully to give saturated or

\footnotetext{
* To whom all correspondence should be addressed.
} 
conjugated polythioesters in high yields. However, the polymers were hardly soluble in conventional organic solvents. The insolubleness may be caused by a cross-linked structure of the polymers due to a homosequence of DVB or DEB. The reactivity of carbothioic acid group may be low.

In the present paper, the authors made a kinetic investigation of the addition reaction of thiobenzoic acid (TBA) to styrene (St) or ethynylbenzene (EB) (eq 1 and 2) as a model of polyaddition of BDTC to DVB or DEB.

$$
\begin{gathered}
\phi \mathrm{CH}=\mathrm{CH}_{2}+\phi \underset{\mathrm{O}}{\mathrm{CSH}} \rightarrow \phi \mathrm{CH}_{2} \mathrm{CH}_{2} \mathrm{SC}_{\Pi}^{\mathrm{O}} \phi \\
\phi \mathrm{C} \equiv \mathrm{CH}+\phi \underset{\|}{\mathrm{CSH}} \rightarrow \phi \mathrm{CH}=\underset{\mathrm{O}}{\mathrm{CHSC}} \phi
\end{gathered}
$$

The rate-determining step and substituent effect on the addition reactions were determined and compared with those on the addition reaction of thiophenol (TP) to $\mathrm{St}^{7}$ or EB. ${ }^{8,9}$

\section{EXPERIMENTAL}

\section{Reagents}

$p$-Methoxy, $p$-methyl, and $p$-chloro TBAs were prepared by the modified synthetic method for TBA ${ }^{10}$ as follows: a solution of $25 \mathrm{~g}(0.15 \mathrm{~mol})$ of $p$-methoxybenzoylchloride (Aldrich Chem. Co.) in $75 \mathrm{ml}$ of anhydrous diethyl ether was added dropwise to an well stirred suspension of $25 \mathrm{~g}(0.44 \mathrm{~mol})$ of $\mathrm{NaSH}$ in $135 \mathrm{ml}$ of anhydrous ethanol at $0-5^{\circ} \mathrm{C}$ under a nitrogen atmosphere. $\mathrm{NaSH}$ was dried over conc sulfuric acid in vacuo overnight. The reaction mixture was stirred at room temperature for $3 \mathrm{~h}$. Approximately $100 \mathrm{ml}$ of water were added to the mixture and the products were dissolved in the water layer. The water layer was extracted five times with $50 \mathrm{ml}$ diethyl ether to remove the by-product ethyl $p$-methoxybenzoate. $20 \mathrm{ml}$ of $6 \mathrm{~N}$ hydrochloric acid were added slowly to the mixture on an ice bath. The precipitate was recovered by filtration, washed with water, and dried over anhydrous calcium chloride in vacuo overnight. The crude product was purified by recrystallization from hexane, yield $70 \%, \mathrm{mp}$ 79.9-81.1 ${ }^{\circ} \mathrm{C}$ (lit. $81-83^{\circ} \mathrm{C}^{11}$ ). $p$-Methyl and $p$-chloro TBAs were prepared from $p$-methyl and $p$-chlorobenzoylchlorides (Tokyo Chem. Ind. Co., Ltd.) in the similar manner [ $p$-Me: yield $71 \%$, mp $40.9-42.2^{\circ} \mathrm{C}$ (lit. $40-42^{\circ} \mathrm{C}^{11}$ ); p-Cl: yield $72 \%, \mathrm{mp} 69.7-70.8^{\circ} \mathrm{C}$ (lit. $70-$ $\left.\left.71^{\circ} \mathrm{C}^{12}\right)\right]$.

TBA and TP were provided by Sumitomo Seika Chem. Co., and $p$-chloro St was supplied by Hokko Chem. Ind. Co., Ltd. p-Methyl, $p$-methoxy Sts, ${ }^{13-15} p$-chloro, ${ }^{16} p$-methyl, and $p$-methoxy $\mathrm{EBs}^{17}$ were synthesized according to the literature. St (Wako Pure Chem. Ind., Ltd.) and EB (Tokyo Chem. Ind. Co., Ltd.) were obtained commercially. All reagents except $p$-substituted TBAs were purified more than two times by vacuum distillation under a nitrogen atmosphere before use. Benzene (Wako Pure Chem. Ind., Ltd.) as a reaction solvent was distilled over calcium hydride and degassed by vacuum for $20 \mathrm{~min}$ before use to exclude oxygen. 2,2'-Azobisisobutyronitrile (AIBN: Wako Pure Chem. Ind., Ltd.) was used as received. Nitrogen gas was purified as shown in the previous paper. ${ }^{7}$

\section{Addition Reaction}

In a $50 \mathrm{ml}$ round bottom flask equipped with a three-way stopcock, each benzene solution of AIBN, Sts, and TBAs was charged by a hypodermic syringe under a nitrogen atmosphere. The reaction was initiated by introducing the reaction flask into a water bath at $60^{\circ} \mathrm{C}$. The course of the reaction was monitored every $15-20$ min by measurements of the adduct concentration by GC using 1-phenyl-2-phenylthioethane $\left(\phi \mathrm{CH}_{2} \mathrm{CH}_{2} \mathrm{~S} \phi\right)$ as an external reference.

\section{Measurement}

Gas chromatograms were made on a Shimadzu GC-4BMPF equipped with a flame ionization detector (FID) and a Silicone GE 
SE-30 column $(2 \mathrm{~m})$ at $250^{\circ} \mathrm{C}$ using a nitrogen carrier gas. ${ }^{1} \mathrm{H}$ NMR spectra were obtained with a JEOL PMX-60si spectrometer in $\mathrm{CDCl}_{3}$ with tetramethylsilane as an internal standard. IR spectra were recorded on a Hitachi 260-50 spectrophotometer by a liquid film method. Sulfur content of the adducts was determined by the modified Schoeniger's method. ${ }^{18}$

\section{RESULTS AND DISCUSSION}

\section{Addition Reactions and Reaction Mechanism}

The addition reactions of TBA to St or EB occurred without an induction period and were completed at $60^{\circ} \mathrm{C}$ for $5 \mathrm{~h}$. The main products were confirmed to be the $1: 1$ adducts of TBA and St or EB with the antiMarkownikoff's structure by ${ }^{1} \mathrm{H}$ NMR and IR analyses. Appreciable amounts of byproducts with higher molecular weight than the adducts were confirmed. The by-products may be oligomers containing more than one St or EB unit, formed by the addition of the intermediate carbon radical to $\mathrm{St}$ or $\mathrm{EB}$ instead of the hydrogen transfer from TBA. In the polyaddition of BDTC to DVB or DEB, such homoaddition is thought to cause a cross-linkage of the polymers.

The authors investigated the controlling factor for the addition reactions by a kinetic method. The addition reaction of TBA to St or EB is considered to proceed in a radical chain mechanism, similar to the addition reaction of $\mathrm{TP}$ to $\mathrm{St}^{7}$ or $\mathrm{EB}{ }^{8}$ Elementary reactions in TBA and St are as follows;

Initiation Reaction

$$
\begin{gathered}
\operatorname{AIBN} \stackrel{k_{\mathrm{d}}}{\longrightarrow} 2 \dot{\mathrm{R}}+\mathrm{N}_{2} \\
\dot{\mathrm{R}}+\phi \underset{\|}{\mathrm{CSH}} \stackrel{k_{\mathrm{i}}}{\longrightarrow} \phi \underset{\prod}{\mathrm{C}} \dot{\mathrm{S}}+\mathrm{RH} \\
\\
\dot{\mathrm{S}}
\end{gathered}
$$

Addition Reaction

Dissociation Reaction

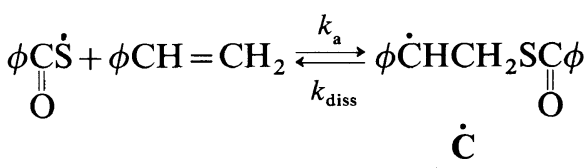

Chain Transfer Reaction

$$
\dot{\mathbf{C}}+\phi \underset{\Pi}{\mathrm{CS}} \underset{\mathrm{O}}{\stackrel{k_{\mathrm{tr}}}{\longrightarrow}} \phi \mathrm{CH}_{2} \mathrm{CH}_{2} \mathrm{SC} \phi+\underset{\|}{\mathrm{O}} \stackrel{\mathrm{O}}{\mathrm{C}} \dot{\mathrm{S}}
$$

$\mathbf{A}$

Termination Reaction

$$
\begin{array}{r}
2 \phi \underset{\text { II }}{\mathrm{C}} \stackrel{k_{4}}{\longrightarrow} \mathrm{T}_{4} \\
\dot{\mathbf{C}}+\phi \underset{\prod}{\mathrm{C}} \dot{\mathrm{S}} \stackrel{k_{5}}{\longrightarrow} \mathrm{T}_{5} \\
2 \dot{\mathbf{C}} \stackrel{k_{6}}{\longrightarrow} \mathrm{T}_{6}
\end{array}
$$

Tautomerism

$$
\begin{aligned}
& \phi \underset{\mathrm{O}}{\mathrm{CSH}} \underset{k_{\mathrm{t} 2}}{\stackrel{k_{\mathrm{t} 1}}{\rightleftarrows}} \phi \underset{\mathrm{S}}{\mathrm{COOH}} \\
& \phi \underset{\text { CI }}{\stackrel{k_{\mathrm{t} 3}}{\rightleftarrows}} \phi \underset{\|}{k_{\mathrm{t} 4}} \dot{\mathrm{O}} \\
& \dot{\mathbf{S}} \quad \dot{\mathbf{O}}
\end{aligned}
$$

where $k_{\mathrm{d}}, k_{\mathrm{i}}, \cdots, k_{\mathrm{t} 4}$ are reaction rate constants for the elementary reactions. The dissociation of $\dot{\mathbf{C}}$ to $\mathrm{St}$ and benzoylthiyl radical $\dot{\mathbf{S}}$ may be negligible because of the resonance stabilization of $\dot{\mathbf{C}} .^{19}$ The tautomerism of TBA and $\dot{\mathbf{S}}$ as shown in eq 7 and 8 is also neglected, since the tautomeric equilibrium of TBA shifted extremely toward the thiol form. ${ }^{20}$ Surely, there was no $\mathrm{C}=\mathrm{S}$ absorption ${ }^{21}$ at $1240 \mathrm{~cm}^{-1}$ in the IR spectrum of the addition products of TBA to $\mathrm{St}$ or $\mathrm{EB}$. Other side reactions are neglected for convenience. As mentioned in the previous paper, ${ }^{7,8}$ the overall reaction 

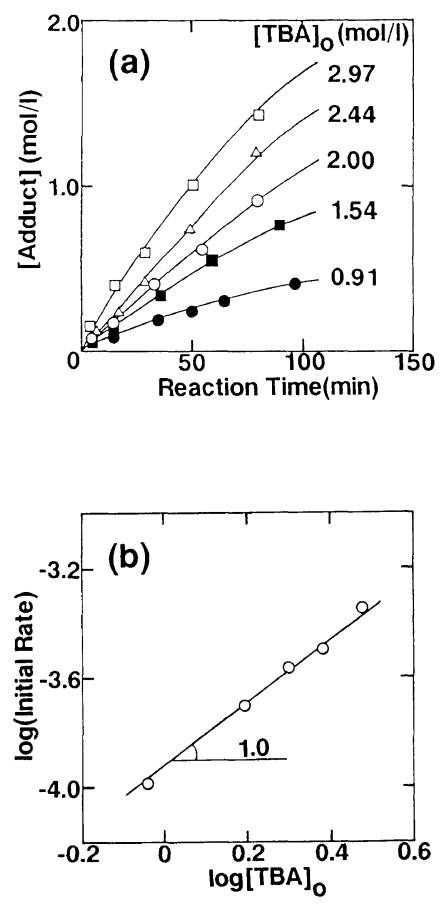

Figure 1. Time-conversion curves (a) of adduct formation with $\mathrm{St}$ by changing the initial concentration of TBA, and reaction order (b) of TBA at $60^{\circ} \mathrm{C}$ in benzene under a nitrogen atmosphere. $[\mathrm{St}]_{0}=2.0 \mathrm{~mol} \mathrm{l}^{-1}$; $[\mathrm{AIBN}]_{0}=$ $2.0 \times 10^{-2} \mathrm{moll}^{-1}$; ext. ref for $\mathrm{GC}, \phi \mathrm{CH}_{2} \mathrm{CH}_{2} \mathrm{~S} \phi$.

rate was expressed by two simple equations. That is, assuming that the rate-determining step is the addition step $\left(k_{\mathrm{a}} \ll k_{\mathrm{tr}}\right)$ or the chain transfer step $\left(k_{\mathrm{a}} \gg k_{\mathrm{tr}}\right)$, the rate equation can be simplified as eq 9 or 10 , respectively.

$$
\begin{array}{cc}
\text { Rate }=w k_{\mathrm{a}}[\mathrm{St} \text { or } \mathrm{EB}]^{1}[\mathrm{AIBN}]^{0.5} \quad\left(k_{\mathrm{a}} \ll k_{\mathrm{tr}}\right) \\
\text { Rate }=w^{\prime} k_{\mathrm{tr}}[\mathrm{TBA}]^{1}[\mathrm{AIBN}]^{0.5} \quad\left(k_{\mathrm{a}} \gg k_{\mathrm{tr}}\right)
\end{array}
$$

where $w$ and $w^{\prime}$ are constant.

\section{Reaction Order and Rate-Determining Step}

Reaction orders of the addition reaction of TBA to St or EB were estimated by a similar method to that described in the previous paper. ${ }^{7,8}$ Typical time-conversion curves and the reaction order for the ad-
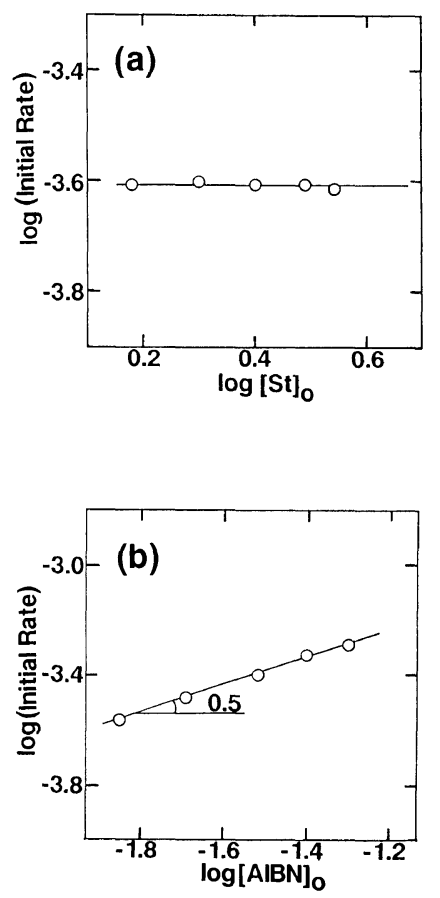

Figure 2. Reaction order of St (a) and AIBN (b) in addition reactions of TBA to St at $60^{\circ} \mathrm{C}$ in benzene under a nitrogen atmosphere. $[\mathrm{TBA}]_{0}=2.0 \mathrm{~mol}^{-1}$; ext. ref for $\mathrm{GC}, \phi \mathrm{CH}_{2} \mathrm{CH}_{2} \mathrm{~S} \phi$ : (a) $[\mathrm{St}]_{0}=1.52-3.45 \mathrm{~mol} \mathrm{l}^{-1}$; $[\mathrm{AIBN}]_{0}=2.0 \times 10^{-2} \mathrm{moll}^{-1}$. (b) $[\mathrm{St}]_{0}=2.0 \mathrm{~mol} \mathrm{1}^{-1}$; $[\mathrm{AIBN}]_{0}=1.5-5.0 \times 10^{-2} \mathrm{~mol} \mathrm{l}^{-1}$.

dition reaction with $\mathrm{St}$ are illustrated in Figures 1 and 2. The addition rate depends on the first order of TBA concentration, 0.5 order of AIBN concentration, and is independent of the St concentration as expressed in eq 11 ;

$$
\text { Rate }(\mathrm{St})=k[\mathrm{TBA}]^{1}[\mathrm{AIBN}]^{0.5}
$$

where $k$ is the overall reaction rate constant in benzene at $60^{\circ} \mathrm{C}$.

On the other hand as shown in Figures 3 and 4 , the addition reaction of TBA to EB can be expressed as the first order of TBA, 0.5 order of AIBN, and is independent of the EB concentration; i.e., the rate equation is expressed by the same eq 11 as for the reaction with $\mathrm{St}$. The experimental rate eq 11 coincides with the theoretical eq 10 , indicating that the rate-determining step of 

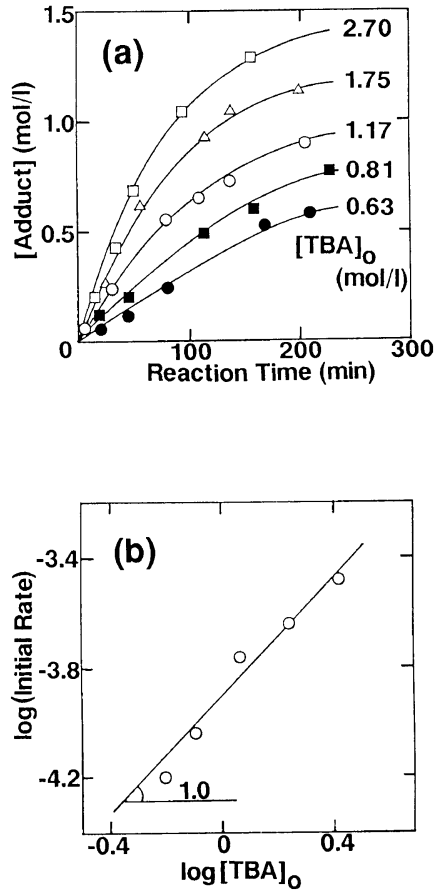

Figure 3. Time-conversion curves (a) of adduct formation with $\mathrm{EB}$ by changing the initial concentration of TBA, and reaction order (b) of TBA at $60^{\circ} \mathrm{C}$ in benzene under a nitrogen atmosphere. $[\mathrm{EB}]_{0}=1.8 \mathrm{~mol} \mathrm{1}^{-1}$; $[\mathrm{AIBN}]_{0}=$ $7.2 \times 10^{-2} \mathrm{~mol} \mathrm{l}^{-1}$; ext. ref for $\mathrm{GC}, \phi \mathrm{CH}_{2} \mathrm{CH}_{2} \mathrm{~S} \phi$.

both reactions is the chain transfer step between each intermediate carbon radical and TBA.

\section{Addition Reactions of TBAs to Sts or EBs}

The addition reactions of TBAs to Sts or EBs were carried out at $60^{\circ} \mathrm{C}$ in benzene under a nitrogen atmosphere. $p$-Chloro, $p$ methyl, and $p$-methoxy derivatives of TBA, St, and EB were used. Using a combination of the above reagents, the addition reactions were completed within 1 day and the adducts were obtained in $68-97 \%$ yields as shown in Table I, confirmed by GC analysis. The ${ }^{1} \mathrm{H}$ NMR spectra showed methylene protons at $2.7-3.4 \mathrm{ppm}$ in the case of Sts. In the ${ }^{1} \mathrm{H}$ NMR spectra of the adducts of TBAs and EBs, the vinylene protons at $6.6-7.0 \mathrm{ppm}$ were confirmed, but signals were partially

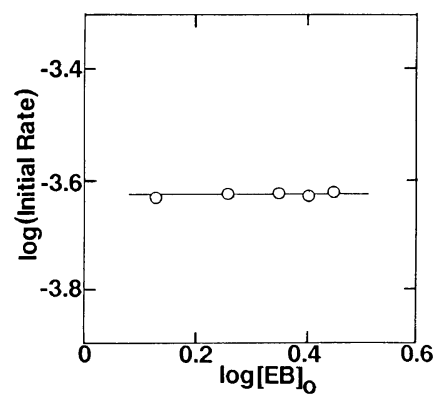

Figure 4. Reaction order of $\mathrm{EB}$ in addition reactions of TBA to $\mathrm{EB}$ at $60^{\circ} \mathrm{C}$ in benzene under a nitrogen atmosphere. $[\mathrm{TBA}]_{0}=1.8 \mathrm{~mol} \mathrm{l}^{-1} ;[\mathrm{EB}]_{0}=1.35$ $2.79 \mathrm{moll}^{-1}$; $[\mathrm{AIBN}]_{0}=7.2 \times 10^{-2} \mathrm{~mol}^{-1}$; ext. ref for $\mathrm{GC}, \phi \mathrm{CH}_{2} \mathrm{CH}_{2} \mathrm{~S} \phi$.

diffused with those of the benzene ring protons. Methyl and vinylidene protons due to Markownikoff's addition to Sts or EBs, respectively, were not confirmed. That is, all adducts were found to have the antiMarkownikoff's structure.

\section{Substituent Effects on the Chain Transfer \\ Step}

Substituent effects on the chain transfer step between the $p$-X substituted intermediate carbon radicals $\left[\mathrm{X}-\phi \dot{\mathrm{C}} \mathrm{HCH}_{2} \mathrm{SC}(=\mathrm{O}) \phi\right.$ or $\mathrm{X}-\phi \dot{\mathrm{C}}=\mathrm{CHSC}(=\mathrm{O}) \phi]$ and $p$-Y substituted TBAs were evaluated in Sts and EBs. These substituent effects will give important informations on the transition state of the rate-determining step in the addition reactions.

In the case of Sts, the relative rates, $k_{\mathrm{tr}}^{\mathbf{X}} / k_{\mathrm{tr}}^{\mathrm{H}}$, of the chain transfer step with $p$-X intermediate carbon radicals were calculated by $R^{\mathrm{X}} / R^{\mathrm{H}}\left(k_{6}^{\mathrm{H}} / k_{6}^{\mathrm{X}}\right)^{0.5}$, where $R^{\mathrm{X}}$ and $R^{\mathrm{H}}$ are the overall addition rates of TBA to $p$-X substituted Sts and to St, respectively, and $k_{6}^{\mathbf{X}}$ and $k_{6}^{\mathrm{H}}$ mean termination reaction constants of the $p-\mathrm{X}$ and the $p-\mathrm{H}$ intermediate carbon radicals, respectively. The overall addition rates, $R^{\mathrm{X}}$ and $R^{\mathrm{H}}$, were determined individually by first order plots on the adduct formation of TBA to $p$-X Sts. The authors assumed that the termination reaction con- 
Table I. Synthesis of adducts with $p-\mathrm{Y}$ TBAs and $p-\mathrm{X} \mathrm{Sts} \mathrm{s}^{\mathrm{a}}$ or $\mathrm{EBs}^{\mathrm{b}}$ at $60^{\circ} \mathrm{C}$ in benzene for 1 day under a nitrogen atmosphere

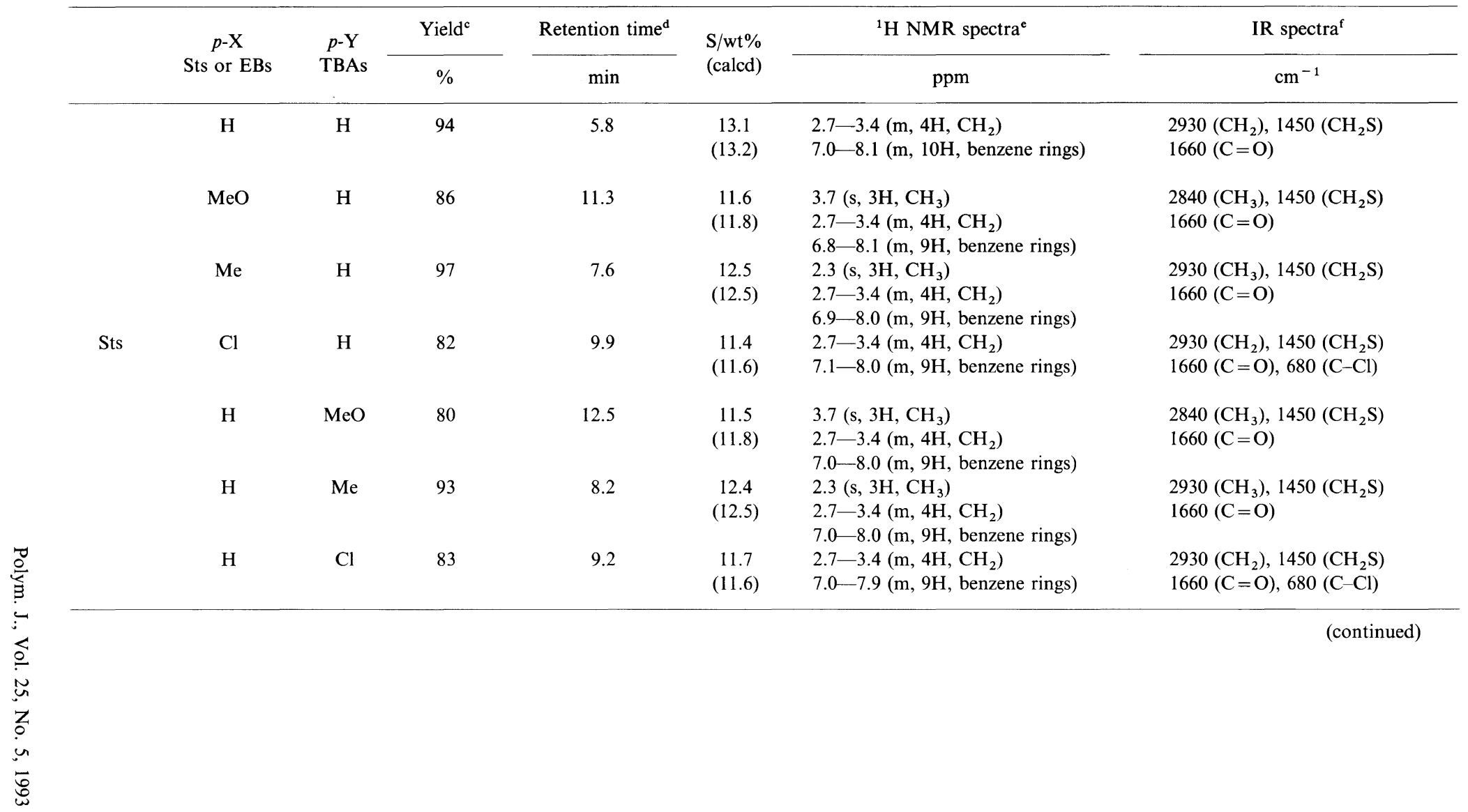


Table I. (Continued)

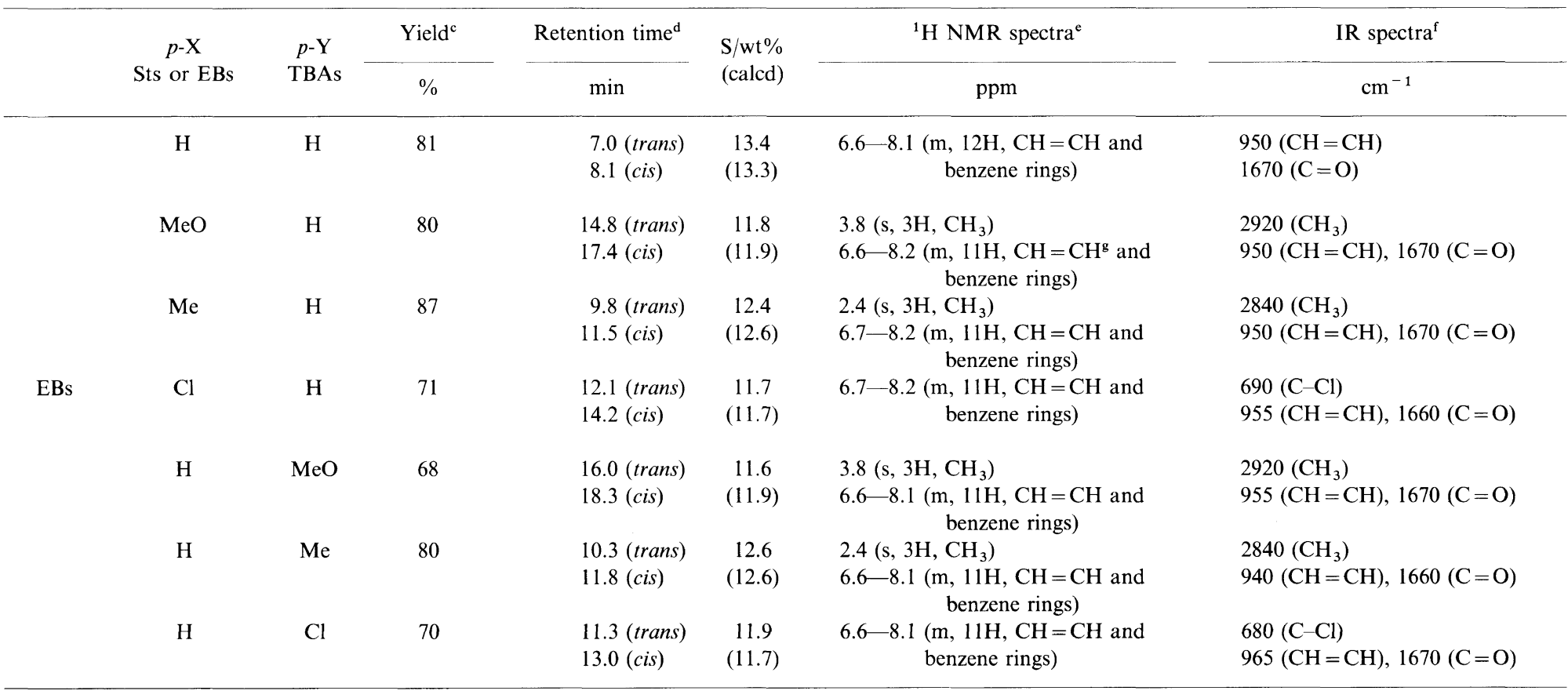

${ }^{\mathrm{a}}[\mathrm{X}-\mathrm{Sts}]_{0}=[\mathrm{Y}-\mathrm{TBAs}]_{0}=2.0 \mathrm{~mol} \mathrm{l}^{-1} ;[\mathrm{AIBN}]_{0}=2.0 \times 10^{-2} \mathrm{~mol} \mathrm{l}^{-1}$.

b $[\mathrm{X}-\mathrm{EBs}]_{0}=[\mathrm{Y}-\mathrm{TBAs}]_{0}=2.0 \mathrm{~mol}^{-1} ;[\mathrm{AIBN}]_{0}=7.2 \times 10^{-2} \mathrm{~mol} \mathrm{l}^{-1}$.

c By GC analysis.

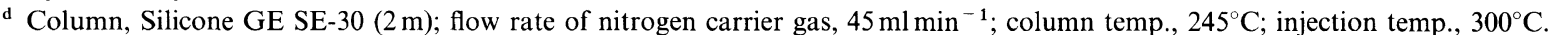

e Solv.: $\mathrm{CDCl}_{3}$, ref: TMS

Liquid film method.

g $6.77,7.04(\mathrm{ABq}$, cis $\mathrm{CH}=\mathrm{CH}), J_{\text {cis } \mathrm{CH}=\mathrm{CH}}=11.0 \mathrm{~Hz} ; 6.79,7.25(\mathrm{ABq}$, trans $\mathrm{CH}=\mathrm{CH}), J_{\text {trans } \mathrm{CH}=\mathrm{CH}}=16.4 \mathrm{~Hz}$; confirmed by JEOL JNM-EX400 spectrometer (400 MHz). 
stants, $k_{6}^{\mathbf{X}}$ and $k_{6}^{\mathrm{H}}$, are equal to those of corresponding polystyryl radicals ${ }^{22}$ as mentioned in the addition reaction of TP to $\mathrm{St},{ }^{7}$ since the intermediate carbon radicals $\left[\mathrm{X}-\phi \dot{\mathrm{C}} \mathrm{HCH}_{2} \mathrm{SC}(=\mathrm{O}) \phi\right]$ in this addition reaction are almost similar to those $\left(\mathrm{X}-\phi \dot{\mathrm{C}} \mathrm{HCH}_{2} \sim\right)$ in the polymerization of $p$-X Sts. On the other hand, the relative rates, $k_{\mathrm{tr}}^{\mathrm{Y}} / k_{\mathrm{tr}}^{\mathrm{H}}$, of the chain transfer step with $p$-Y TBAs were determined by the competitive reaction of TBA and $p$-Y TBAs to St. The theoretical rate equation was similar to that in the previous paper. ${ }^{9}$

In the case of EBs, the relative rates, $k_{\mathrm{tr}}^{\mathbf{X}} / k_{\mathrm{tr}}^{\mathbf{H}}$, of the chain transfer step were tentatively calculated assuming that the termination reaction constant $k_{6}^{\mathrm{X}}$ is equal to $k_{6}^{\mathrm{H}}$; that is, $k_{\mathrm{tr}}^{\mathbf{X}} / k_{\mathrm{tr}}^{\mathrm{H}}$ were calculated by $R^{\mathrm{X}} / R^{\mathrm{H}} \cdot k_{\mathrm{tr}}^{\mathbf{Y}} / k_{\mathrm{tr}}^{\mathrm{H}}$ were determined by the same method as for Sts.

The relative rates, $k_{\mathrm{tr}}^{\mathbf{X}} / k_{\mathrm{tr}}^{\mathrm{H}}$ and $k_{\mathrm{tr}}^{\mathrm{Y}} / k_{\mathrm{tr}}^{\mathrm{H}}$, in both the St and EB systems are summarized in Table II. In both Sts and EBs, $k_{\mathrm{tr}}^{\mathbf{X}} / k_{\mathrm{tr}}^{\mathbf{H}}$ decreases and $k_{\mathrm{tr}}^{\mathrm{Y}} / k_{\mathrm{tr}}^{\mathrm{H}}$ increases in the order of $\mathrm{MeO}, \mathrm{Me}, \mathrm{H}$, and $\mathrm{Cl}$ except for the addition

Table II. Relative rates $\left(k_{\mathrm{tr}}^{\mathrm{X}} / k_{\mathrm{tr}}^{\mathrm{H}}\right.$ and $\left.k_{\mathrm{tr}}^{\mathrm{Y}} / k_{\mathrm{tr}}^{\mathrm{H}}\right)$ of chain transfer step in addition reactions of $p$-Y TBAs to $p$-X Sts or $p$-X EBs at $60^{\circ} \mathrm{C}$ in benzene under a nitrogen atmosphere

\begin{tabular}{|c|c|c|c|c|}
\hline \multirow{2}{*}{$\begin{array}{r}p-\mathrm{XC} \text { 's or } \\
p-\mathrm{Y} \text { TBAs }\end{array}$} & \multicolumn{2}{|c|}{ St } & \multicolumn{2}{|c|}{ EB } \\
\hline & $k_{\mathrm{tr}}^{\mathbf{X}} / k_{\mathrm{tr}}^{\mathrm{H}^{\mathrm{a}}}$ & $k_{\mathrm{tr}}^{\mathrm{Y}} / k_{\mathrm{tr}}^{\mathrm{H}^{\mathrm{b}}}$ & $k_{\mathrm{tr}}^{\mathbf{X}} / k_{\mathrm{tr}}^{\mathbf{H}^{\mathrm{c}}}$ & $k_{\mathrm{tr}}^{\mathrm{Y}} / k_{\mathrm{tr}}^{\mathrm{H}^{\mathrm{d}}}$ \\
\hline $\mathrm{MeO}$ & 3.23 & 0.87 & 1.61 & 0.72 \\
\hline $\mathrm{Me}$ & 1.84 & 0.90 & 1.34 & 0.91 \\
\hline $\mathrm{H}$ & 1.00 & 1.00 & 1.00 & 1.00 \\
\hline $\mathrm{Cl}$ & 0.58 & 1.22 & 0.36 & 0.97 \\
\hline
\end{tabular}

a $[\mathrm{X}-\mathrm{Sts}]_{0}=[\mathrm{TBA}]_{0}=2.0 \mathrm{~mol} \mathrm{l}^{-1} ;[\mathrm{AIBN}]_{0}=2.0 \times$ $10^{-2} \mathrm{~mol} \mathrm{l}^{-1} ; k_{\mathrm{tr}}^{\mathbf{X}} / k_{\mathrm{tr}}^{\mathrm{H}}=R^{\mathrm{X}} / R^{\mathrm{H}}\left(k_{6}^{\mathrm{H}} / k_{6}^{\mathrm{X}}\right)^{0.5} ; R^{\mathrm{MeO}} / R^{\mathrm{H}}=$ $1.80, \quad R^{\mathrm{Me}} / R^{\mathrm{H}}=1.44, \quad R^{\mathrm{Cl}} / R^{\mathrm{H}}=0.49, \quad k_{6}^{\mathrm{H}} / k_{6}^{\mathrm{MeO}}=3.22$, $k_{6}^{\mathrm{H}} / k_{6}^{\mathrm{Me}}=1.64, k_{6}^{\mathrm{H}} / k_{6}^{\mathrm{Cl}}=1.41$.

b $[\mathrm{Sts}]_{0}=2.0 \mathrm{moll}^{-1} ;[\mathrm{TBA}]_{0}=[\mathrm{Y}-\mathrm{TBAs}]_{0}=1.0$ $\mathrm{moll}^{-1} ;[\mathrm{AIBN}]_{0}=2.0 \times 10^{-2} \mathrm{moll}^{-1}$.

c $[\mathrm{X}-\mathrm{EBs}]_{0}=[\mathrm{TBA}]_{0}=2.0 \mathrm{~mol} \mathrm{1}^{-1} ;[\mathrm{AIBN}]_{0}=7.2 \times$ $10^{-2} \mathrm{~mol} \mathrm{l}^{-1} ; k_{\mathrm{tr}}^{\mathrm{X}} / k_{\mathrm{tr}}^{\mathrm{H}} \simeq R^{\mathrm{X}} / R^{\mathrm{H}}$.

${ }^{\mathrm{d}}[\mathrm{EBs}]_{0}=2.0 \mathrm{moll}^{-1} ;[\mathrm{TBA}]_{0}=[\mathrm{Y}-\mathrm{TBAs}]_{0}=1.0$ $\mathrm{moll}^{-1} ;[\mathrm{AIBN}]_{0}=7.2 \times 10^{-2} \mathrm{moll}^{-1}$. reaction of $p-\mathrm{Cl}$ TBA to $\mathrm{EB}$. That is, the electron-donating $p$-X group on the intermediate carbon radical increases the reaction rate. In contrast, the electron-donating $p$-Y group on TBA decreases the reaction rate.

These relative rates were correlated by Hammett's eq 12 and $13 .^{23}$

$$
\begin{gathered}
\log \left(k / k_{0}\right)=\rho \sigma \\
\log \left(k / k_{0}\right)=\rho \sigma+\gamma E_{\mathbf{R}}
\end{gathered}
$$

In eq $13, \sigma$ and $E_{\mathrm{R}}$ are polar and resonance substituent constants, $\rho$ and $\gamma$ are reaction constants giving the susceptibility of the polar and resonance effects caused by the substituent, respectively. That is, $\rho \sigma$ shows a polar term
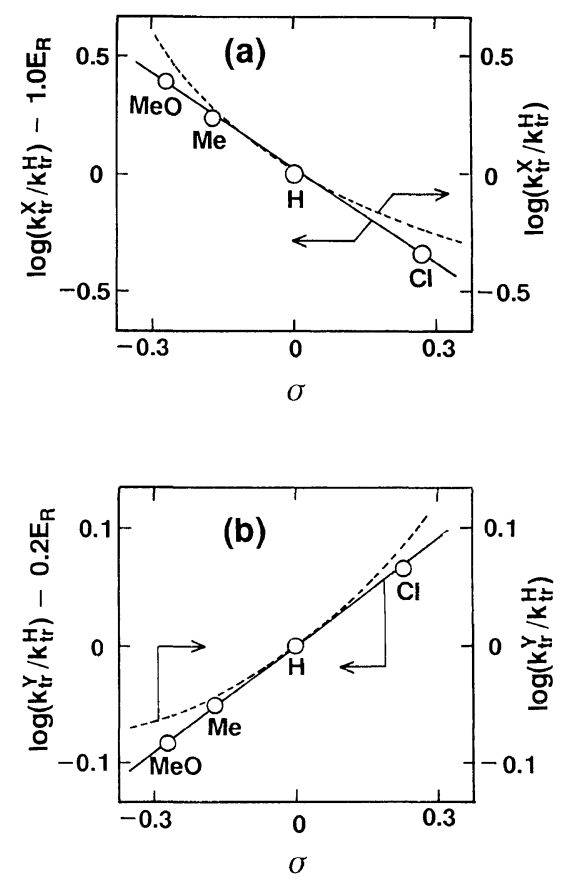

Figure 5. Hammett plots for chain transfer step between $p$-X intermediate carbon radicals $\left[\mathrm{X}-\phi \dot{\mathrm{C}} \mathrm{HCH}_{2} \mathrm{SC}(=\mathrm{O}) \phi\right]$ and TBA (a), and between $p$ - $\mathrm{H}$ intermediate carbon radical $\left[\phi \dot{\mathrm{C}} \mathrm{HCH}_{2} \mathrm{SC}(=\mathrm{O}) \phi\right]$ and $p$-Y TBAs (b) at $60^{\circ} \mathrm{C}$ in benzene under a nitrogen atmosphere. Broken (----) and solid lines (-) were correlated by eq 12 and 13 , respectively. (a) $[\mathrm{X}-\mathrm{Sts}]_{0}=[\mathrm{TBA}]_{0}=2.0 \mathrm{~mol} \mathrm{l}^{-1}$; $[\mathrm{AIBN}]_{0}=2.0 \times 10^{-2} \mathrm{~mol} \mathrm{I}^{-1} ; \log \left(k_{\mathrm{tr}}^{\mathrm{X}} / k_{\mathrm{tr}}^{\mathrm{H}}\right)=-1.48 \sigma+$ $1.0 E_{\mathrm{R}}$. (b) $[\mathrm{St}]_{0}=2.0 \mathrm{moll}^{-1} ; \quad[\mathrm{TBA}]_{0}=[\mathrm{Y}-\mathrm{TBAs}]_{0}=$ $1.0 \mathrm{~mol} \mathrm{l}^{-1} ;[\mathrm{AIBN}]_{0}=2.0 \times 10^{-2} \mathrm{~mol} \mathrm{l}^{-1} ; \log \left(k_{\mathrm{tr}}^{\mathrm{Y}} / k_{\mathrm{tr}}^{\mathrm{H}}\right)=$ $0.30 \sigma+0.2 E_{\mathrm{R}}$. 
and $\gamma E_{\mathrm{R}}$ is a resonance term. Figures 5(a) and (b) show the Hammett plot for the chain transfer step between $p$-X intermediate
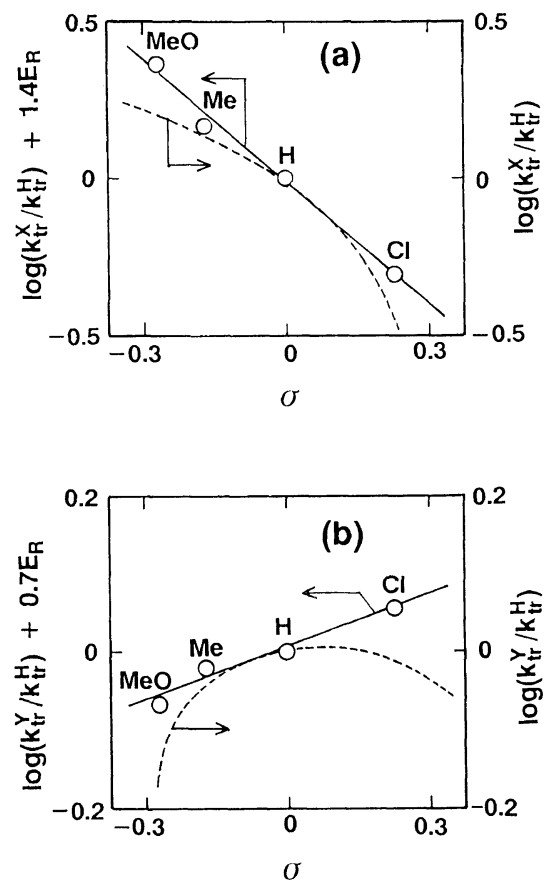

Figure 6. Hammett plots for chain transfer step between $p$-X intermediate carbon radicals $[\mathrm{X}-\phi \dot{\mathrm{C}}=\mathrm{CHSC}(=\mathrm{O}) \phi]$ and TBA (a), and between $p-\mathrm{H}$ intermediate carbon radical $[\phi \dot{\mathrm{C}}=\mathrm{CHSC}(=\mathrm{O}) \phi]$ and $p$-Y TBAs $(\mathrm{b})$ at $60^{\circ} \mathrm{C}$ in benzene under a nitrogen atmosphere. Broken (----) and solid lines (-) were correlated by eq 12 and 13 , respectively. (a) $[\mathrm{X}-\mathrm{EBs}]_{0}=[\mathrm{TBA}]_{0}=2.0 \mathrm{~mol} \mathrm{l}^{-1}$; $[\mathrm{AIBN}]_{0}=7.2 \times 10^{-2} \mathrm{~mol} \mathrm{l}^{-1} ; \log \left(k_{\mathrm{tr}}^{\mathrm{X}} / k_{\mathrm{tr}}^{\mathrm{H}}\right)=-1.35 \sigma-$ $1.4 E_{\mathrm{R}}$. (b) $[\mathrm{EB}]_{0}=2.0 \mathrm{~mol} \mathrm{l}^{-1} ;[\mathrm{TBA}]_{0}=[\mathrm{Y}-\mathrm{TBAs}]_{0}=$ $1.0 \mathrm{~mol} \mathrm{l}^{-1} ;[\mathrm{AIBN}]_{0}=7.2 \times 10^{-2} \mathrm{~mol} \mathrm{l}^{-1} ; \log \left(k_{\mathrm{tr}}^{\mathrm{Y}} / k_{\mathrm{rr}}^{\mathrm{H}}\right)=$ $0.23 \sigma-0.7 E_{\mathrm{R}}$. carbon radicals $\left[\mathrm{X}-\phi \dot{\mathrm{C}} \mathrm{HCH}_{2} \mathrm{SC}(=\mathrm{O}) \phi\right]$ and TBA, or between the $p-\mathrm{H}$ intermediate carbon radical $\left[\phi \dot{\mathrm{C}} \mathrm{HCH}_{2} \mathrm{SC}(=\mathrm{O}) \phi\right]$ and $p$-Y TBAs, respectively, according to eq 12 and 13. Similarly, Figure 6 shows the Hammett correlation of the chain transfer step in the EB system. The broken and solid lines in Figures 5 and 6 were correlated by eq 12 and 13, respectively. Better correlation was obtained with eq 13 rather than eq 12 in all cases. That is, the rate of the addition reaction of TBAs to Sts or EBs is controlled not only by a polar term but also by a resonance term. Similar results have been discussed on the substituent effect of chain transfer reactions between polystyryl radical and cumenes ${ }^{24}$ or toluenes. $^{25}$

The reaction constant, $\rho$, and resonance constant, $\gamma$, obtained by eq 13 from Figures 5 and 6 are summarized in Table III. $\rho$ of the chain transfer step in both Sts $(\rho=-1.48)$ and EBs $(\rho=-1.35)$ are negative and the difference is small, indicating that the transition state of the chain transfer step between the each intermediate carbon radical and TBA is much alike. On the other hand, $\rho$ of the chain transfer step between the $p-\mathrm{H}$ intermediate carbon radicals and $p$-Y TBAs (St, $\rho=0.30$; EB, $\rho=0.23$ ) are positive. The rate of the chain transfer step between each intermediate carbon radical and TBAs increases with decreasing electron density of $\mathrm{S}-\mathrm{H}$ bonds on TBAs and with increasing

Table III. Hammett parameters obtained from Figures 5 and 6 for chain transfer step of addition reactions of $p$-Y TBAs to $p$-X Sts and $p$-X EBs

\begin{tabular}{ccc}
$\mathrm{St}$ & $\mathrm{EB}$ \\
$p-\mathrm{X} \dot{\mathrm{C}} \mathrm{s}$ & $\begin{array}{c}\mathrm{X}-\phi \dot{\mathrm{C}} \mathrm{HCH}_{2} \mathrm{SC}(=\mathrm{O}) \phi \\
+\phi \mathrm{C}(=\mathrm{O}) \mathrm{SH}\end{array}$ & $\begin{array}{c}\mathrm{X}-\phi \dot{\mathrm{C}}=\mathrm{CHSC}(=\mathrm{O}) \phi \\
+\phi \mathrm{C}(=\mathrm{O}) \mathrm{SH}\end{array}$ \\
\hline$p-\mathrm{Y}$ TBAs & $-1.48 \sigma+1.0 E_{\mathrm{R}}$ & $-1.35 \sigma-1.4 E_{\mathrm{R}}$ \\
\hline $\begin{array}{c}\phi \dot{\mathrm{C}} \mathrm{HCH}_{2} \mathrm{SC}(=\mathrm{O}) \phi \\
+\mathrm{Y}-\phi \mathrm{C}(=\mathrm{O}) \mathrm{SH}\end{array}$ & $\begin{array}{c}\dot{\mathrm{C}}=\mathrm{CHSC}(=\mathrm{O}) \phi \\
+\mathrm{Y}-\phi \mathrm{C}(=\mathrm{O}) \mathrm{SH}\end{array}$ \\
& $0.30 \sigma+0.2 E_{\mathrm{R}}$ & $0.23 \sigma-0.7 E_{\mathrm{R}}$
\end{tabular}


the electron density of intermediate carbon radicals. That is, it is assumed that the electron is somewhat transferred from the intermediate carbon radicals to TBA in the transition state, and cleavage of the $\mathrm{S}-\mathrm{H}$ bond occurs. Such an idea has been proposed by Imoto $^{26}$ for the substituent effect on chain transfer reactions between polystyryl radical and $p$-substituted cumenes, in which the reaction constant $\rho$ is positive. Consequently, a perturbation between SOMO of each intermediate carbon radical and LUMO of TBA in the transition state is the important controlling factor for the reactivity in the adduct formation of TBA and St or EB. It was also found that both the intermediate carbon radicals are nucleophilic, and the nucleophilicity of the carbon radicals is similar, since $\rho$ of both systems is almost equal. The absolute values of $\rho$ and $\gamma$ for the $p$-X radicals were larger than those for the $p$-Y TBAs. The inductive and resonance effects ( $I-$ and $R$-effects) by the $p$-X substituent on the intermediate carbon radicals directly affect the reaction reactivity, while those by the $p-Y$ substituent on TBAs have less influence on reactivity. That is, the $I$ - and $R$-effects by the $p$-Y substituent on TBAs may be decreased by the carbonyl group. In the substituent effect by $\mathrm{X}$ and $\mathrm{Y}$ substituents, $\gamma$ for Sts $(\mathrm{X}, \gamma=1.0 ; \mathrm{Y}, \gamma=0.2)$ was positive and that for EBs (X, $\gamma=-1.4$; $\mathrm{Y}, \gamma=-0.7)$ was negative. Otsu et al. ${ }^{9,25}$ discusses the sign of $\gamma$. That is, in the $\mathrm{St}$ system the $R$-effect by the $\mathrm{X}$ and the $\mathrm{Y}$ substituents on the radical and TBA, respectively, in the transition state is larger than that in the initial state. In the EB system the $R$-effect by the substituents in the transition state is smaller. The influence of the $R$-effect by the substituent on the reactivity in EBs is found to be slightly larger than that in Sts, because absolute $\gamma$ value in EBs is larger than that in Sts.

Accordingly, the overall reaction rate of the adduct formation of Sts with TBAs increases by introducing a large electrondonating $(+I)$ group into the para position of St or a large $-I$ group into that of TBA, and/or high resonance stabilizing $(+R$ or $-R$ ) group into the para position of $\mathrm{St}$ and TBA. On the other hand, EBs having a large $+I$ group or TBAs containing a large $-I$ group on the para position accelerate addition reactions in EBs, but the resonance effect by the para substituent depresses the reactivity.

\section{Polyaddition Reactivity of BDTC to DVB or $D E B$}

The authors estimated polyaddition reactivity based on the above substituent effect. The polyaddition of BDTC to DVB or DEB can be regarded as the addition reaction of TBA having $p$-COSH group to $\mathrm{St}$ having $p$-vinyl group or EB having $p$-ethynyl group, respectively. $\sigma$ for the $p$-vinyl and $p$-ethynyl substituents have been reported as $-0.08^{27}$ and $0.233,{ }^{28}$ respectively, by $\mathrm{p} K_{\mathrm{a}} . \sigma$ for the $p$-COSH group has not been reported and is thought to be a positive, since $\sigma$ for $p-\mathrm{COOH}$ group is $0.45 .^{29} \gamma$ for the $p$-vinyl, $p$-ethynyl, and $p$-COSH groups are unclear and are expected to be positive, because the $p$-vinyl and p-ethynyl groups on St and EB have a planner and $\pi$-conjugated structure and $p-\mathrm{COSH}$ is the $-R$ group. Accordingly, the polyaddition of BDTC to DVB is much faster than the addition reaction of TBA to St. For example, on assuming tentatively that $\sigma$ for the $p$-COSH group is 0.45 and $\gamma$ for the $p$-vinyl and $p$-COSH groups is 0.11 , which is $\gamma$ for the $p$-methoxy group, ${ }^{23}$ the estimated reactivity of the addition reaction of BDTC to DVB is about twelve times that of TBA to St. However, the polymer was insoluble and had a cross-linked structure formed by the homosequence of DVB as confirmed by hydrolysis of the polymer. ${ }^{5,6}$ To obtain a soluble polymer with a linear structure, it may be necessary to introduce a large $+I$ group and a large $R$ group into 
DVB or a large $-I$ group and a large $R$ group into BDTC.

On the other hand, the polyaddition of BDTC to DEB is expected to be similar to or lower than the addition reaction of TBA to EB in the reactivity due to the substituent groups of $p$-COSH and $p$-ethynyl. That is, the polymer of BDTC and DEB is thought to have many cross-linkages in the polymer chain. In fact, the absorption edge of this polymer in the diffuse reflectance spectrum was at $530 \mathrm{~nm},{ }^{6}$ this being shorter wavelength than that of the polymer of 1,4-benzenedithiol (BDT) and DEB. However, it may be possible to increase the polyaddition reactivity of $\mathrm{DEB}$, since the $+I$ substituent increases the reactivity of DEB.

\section{Substituent Effect on the Addition Step}

The substituent effect on the addition step of benzoylthiyl radical to $p$-X Sts or $p$-X EBs is also examined. The relative rates of the addition step, $k_{\mathrm{a}}^{\mathbf{X}} / k_{\mathrm{a}}^{\mathrm{H}}$, were estimated by the competitive reaction of TBA to $\mathrm{St}$ and $p$-X Sts or to EB and $p$-X EBs. Table IV summarizes the relative rates in both systems. Figure 7 shows the Hammett correlation of the addition step according to eq 12 and 13. Better correlation was obtained with eq 13 rather than eq 12 in both systems. That is, resonance as well as polar effects are

Table IV. Relative rates $\left(k_{\mathrm{a}}^{\mathrm{X}} / k_{\mathrm{a}}^{\mathrm{H}}\right)$ of addition step in addition reactions of TBA to $p$-X Sts or $p$-X EBs at $60^{\circ} \mathrm{C}$ in benzene under a nitrogen atmosphere

\begin{tabular}{lccc}
\hline \multirow{2}{*}{$\begin{array}{c}p \text {-X Sts } \\
\text { or EBs }\end{array}$} & $\mathrm{St}$ & $\mathrm{EB}$ \\
\cline { 2 - 2 } & $k_{\mathrm{a}}^{\mathbf{X}} / k_{\mathrm{a}}^{\mathrm{H}^{\mathbf{a}}}$ & & $k_{\mathrm{a}}^{\mathbf{X}} / k_{\mathbf{a}}^{\mathbf{H}^{\mathbf{b}}}$ \\
\hline $\mathrm{MeO}$ & 1.90 & & 2.34 \\
$\mathrm{Me}$ & 1.44 & & 1.43 \\
$\mathrm{H}$ & 1.00 & & 1.00 \\
$\mathrm{Cl}$ & 0.95 & 0.93
\end{tabular}

${ }^{\mathrm{a}}[\mathrm{X}-\mathrm{Sts}]_{0}=[\mathrm{St}]_{0}=1.0 \mathrm{~mol}^{-1} ;[\mathrm{TBA}]_{0}=2.0 \mathrm{moll}^{-1}$; $[\mathrm{AIBN}]_{0}=2.0 \times 10^{-2} \mathrm{~mol}^{-1}$.

${ }^{\mathrm{b}}[\mathrm{X}-\mathrm{EBs}]_{0}=[\mathrm{EB}]_{0}=1.0 \mathrm{~mol} \mathrm{l}^{-1} ;[\mathrm{TBA}]_{0}=2.0$ $\mathrm{moll}^{-1} ;[\mathrm{AIBN}]_{0}=7.2 \times 10^{-2} \mathrm{~mol} \mathrm{1}^{-1}$. important factors to control the addition step of benzoylthiyl radical to St or EB. The transition states of the addition step with $\mathrm{St}$ and EB are thought to be much alike, since $\rho$ and $\gamma$ are little changed (Sts, $\rho=-0.61$; $\gamma=1.1 ;$ EBs, $\rho=-0.76 ; \gamma=1.5) . \quad \rho$ of the addition step suggests that benzoylthiyl radical is electrophilic, and perturbation between SOMO of the radical and HOMO of St or EB in the transition state is an important controlling factor for the addition step. $\gamma$ of both the systems was positive, indicating that the addition step is accelerated by the $R$-effect of the $p$-X substituent on $\mathrm{St}$ and EB. $\gamma$ of Sts is slightly smaller than that of EBs. This indicates that the addition step with EB is more stabilized by the resonance
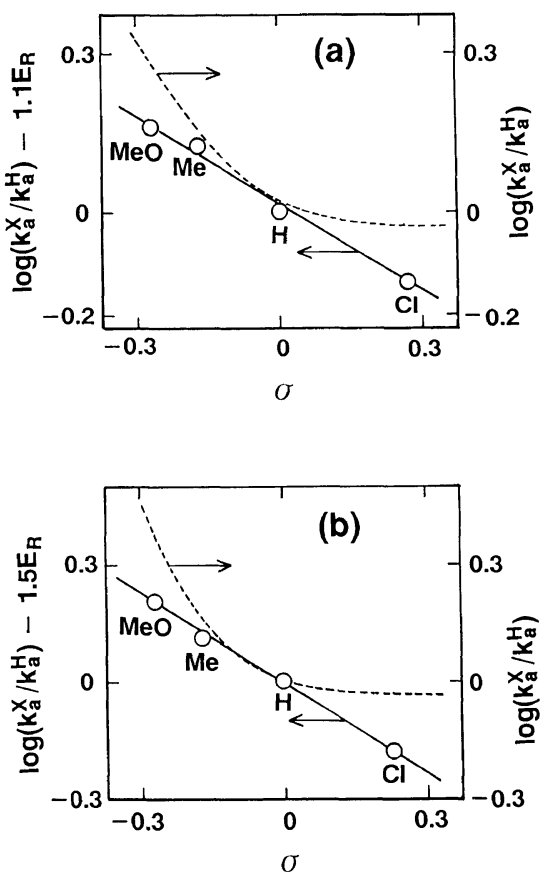

Figure 7. Hammett plots for addition step of benzoylthiyl radical $[\phi \mathrm{C}(=\mathrm{O}) \dot{\mathrm{S}}]$ and $p$-X Sts $(\mathrm{a})$, and $p-\mathrm{X}$ EBs (b) at $60^{\circ} \mathrm{C}$ in benzene under a nitrogen atmosphere. Broken (----) and solid lines (-) were correlated by eq 12 and 13 , respectively. (a) $[\mathrm{X}-\mathrm{Sts}]_{0}=[\mathrm{St}]_{0}=$ $1.0 \mathrm{~mol} \mathrm{l}^{-1} ;[\mathrm{TBA}]_{0}=2.0 \mathrm{~mol} \mathrm{l}^{-1} ;[\mathrm{AIBN}]_{0}=2.0 \times 10^{-2}$ $\mathrm{mol} \mathrm{1}^{-1} ; \log \left(k_{\mathrm{a}}^{\mathbf{X}} / k_{\mathrm{a}}^{\mathrm{H}}\right)=-0.61 \sigma+1.1 E_{\mathrm{R}}$. (b) $[\mathrm{X}-\mathrm{EBs}]_{0}=$ $[\mathrm{EB}]_{0}=1.0 \mathrm{~mol} \mathrm{l}^{-1} ;[\mathrm{TBA}]_{0}=2.0 \mathrm{~mol} \mathrm{l}^{-1} ;[\mathrm{AIBN}]_{0}=$ $7.2 \times 10^{-2} \mathrm{~mol} \mathrm{l}^{-1} ; \log \left(k_{\mathrm{a}}^{\mathrm{X}} / k_{\mathrm{a}}^{\mathrm{H}}\right)=-0.76 \sigma+1.5 E_{\mathrm{R}}$. 
conditions and polymer yields to decrease in the order of BDT-DEB, ${ }^{30}$ BDT-DVB, ${ }^{31}$ BDTC-DVB, ${ }^{5}$ and BDTC-DEB,${ }^{6}$ this being the same as the order in Table V. Here, estimated reactivity does not mean a difference in the absolute rate but apparent and relative values, since the rate-determining step of the addition reaction of TP to EB was different from that of TP-St, TBA-St, and TBA-EB as is mentioned in the next section.

Kinetic Behavior. The kinetic behavior of the addition reactions of TBA-St, TP-St, TBA-EB, and TP-EB is summarized in Table VI. The rate-determining step of the addition reactions is the chain transfer step, except for TP-EB. The rate-determining step of TP-EB is the addition step of the benzenethiyl radical to $\mathrm{EB} .{ }^{8}$ The discrepancy in the ratedetermining step of TP-EB may be caused by $\mathrm{CT}$ interactions between TP and EB as mentioned in the previous paper. ${ }^{9}$ Large CT interaction between TP and EB has been already confirmed, ${ }^{32}$ but in other cases, such large CT interaction was not confirmed in the UV spectra of each reagent mixture.

In the case of the addition step, the transition states are thought to be much alike, since $\rho$ and $\gamma$ of all reactions have the same sign, in which $\rho$ is negative and $\gamma$ is positive. But, absolute $\rho$ values of TBA system are about twice those of the TP system. Imoto et $a .^{22}$ claim that $\rho$ changed greatly by the polarity of the attacking radical controlled by the substituent in the addition reactions of various polystyryl radicals to substituted styrenes. It was also found in the present system that the electrophilicity of the benzoylthiyl radical was larger than that of the benzenethiyl radical to St or EB. $\gamma$ of the TP system are larger than those of TBA system in each addition step to $\mathrm{St}$ or $\mathrm{EB}$, indicating that an activated complex containing TP in the transition state is more resonance-stabilized by the $p$-X substituent than that containing TBA.
In the case of the chain transfer step, the transition states of TBA-St, TBA-EB, and TP-St are also alike. That is, the intermediate carbon radicals of TBA-St, TBAEB, and TP-St are nucleophilic, since $\rho$ is positive. On the other hand, the intermediate carbon radical of TP-EB is electrophilic $(\rho<0)$. This difference may alter the ratedetermining step of the addition reaction of TP to EB. Absolute $\rho$ values of the TBA system are much smaller than those of TP system, indicating that the magnitude of the $\mathrm{S}-\mathrm{H}$ bond cleavage of TBA in the transition state is smaller than that of TP and results for the lower reactivity of TBA than TP to St or EB. Such an idea has been proposed for the substituent effect on hydrogen abstraction of various radicals from substituted toluenes. ${ }^{23}$ Absolute $\gamma$ values of TBAs are also much smaller than those of TPs. The chain transfer step of TPs is more stabilized by the $R$-effect than that of TBAs in the transition state. That is, resonance stabilization by $p$-X and $p$-Y substituents in the transition state may decrease by the carbonyl group.

Consequently, the controlling factor for the radical addition reaction of TP or TBA to $\mathrm{St}$ or EB is perturbation between SOMO of each intermediate carbon radical and LUMO of TBA or TP, except TP-EB. In the case of TP-EB, the controlling factor is perturbation between SOMO of the benzenethiyl radical and HOMO of EB. The differences in reactivities of the each addition reaction may be also clarified by a molecular orbital calculation of the band gap between the perturbation orbitals. This is now being done.

\section{REFERENCES}

1. F. W. Stacey and J. F. Harris, Jr., J. Org. Reactions, 13, 150 (1963).

2. N. H. Koening and D. Swern, J. Am. Chem. Soc., 79, 4235 (1957); N. H. Koening, S. Sasin, and D. 
Swern, J. Org. Chem., 23, 1525 (1958).

3. H. Bader, L. C. Cross, I. Heilbron, and E. R. H. Jones, J. Chem. Soc., 619 (1949); K. Yamagishi, T. Tanaka, and T. Hoshino, Bull. Chem. Soc. Jpn., 30, 455 (1957).

4. C. S. Marvel and A. Kotch, J. Am. Chem. Soc., 73, 1100 (1951); C. S. Marvel and E. A. Kraimann, J. Org. Chem., 18, 707 (1953).

5. E. Kobayashi, M. Kato, S. Aoshima, and J. Furukawa, Polym. Prepr. Jpn., 40, 282 (1991); E. Kobayashi, K. Tendo, S. Aoshima, and J. Furukawa, Polym. Prepr. Jpn., 40, 1803 (1991); Polym. J. to be prepared.

6. E. Kobayashi, K. Tendo, S. Aoshima, and J. Furukawa, Polym. Prepr. Jpn., 41, 1884 (1992).

7. E. Kobayashi, T. Obata, S. Aoshima, and J. Furukawa, Polym. J., 22, 803 (1990).

8. E. Kobayashi, T. Obata, S. Aoshima, and J. Furukawa, Polym. J., 22, 1035 (1990).

9. T. Obata, E. Kobayashi, S. Aoshima, and J. Furukawa, Polym. J., 24, 757 (1992).

10. N. Noble and D. S. Tarbell, Org. Synth., Coll. Vol., IV, 924 (1963).

11. Y. Kobayashi and K. Itabashi, Synthesis, 670 (1985).

12. S. Motoki and M. Bergmann, Ber. Dtsch. Chem. Ges., 53, 975 (1920).

13. R. Adains and C. R. Noller, Org. Synth., Coll. Vol., II, 109 (1955).

14. H. G. France, R. E.Lutz, and R. L. Wayland, J. Am. Chem. Soc., 72, 5511 (1950).

15. C. G. Overberger and H. H. Sounders, Org. Synth., Coll. Vol., III, 204 (1955).
16. A. S. Hay, J. Org. Chem., 25, 637 (1960).

17. D. J. Dawson, J. D. Frazier, P. J. Brock, and R. J. Twieg, Polym. Mater. Sci. Eng., 55, 401 (1986).

18. W. Schoeniger, Mikrochim. Acta, 123 (1955).

19. R. H. Pallen and C. Sivertz, Can. J. Chem., 35, 723 (1957).

20. Yu. N. Sheinker, S. T. Ioffe, and M. I. Kabachnik, Izvest. Akad. Nauk S.S.S.R., Otdel. Khim. Nauk., 1571 (1960); Chem. Abstr., 55, 9323 (1961).

21. D. H. R. Barton, C. Chavis, M. K. Kaloustian, P. D. Magnus, G. A. Poulton, and P. J. West, J. Chem. Soc., Perkin Trans. 1, 1571 (1973).

22. M. Imoto, M. Kinoshita, and M. Nishizaki, Makromol. Chem., 86, 217 (1965).

23. T. Yamamoto and T. Otsu, J. Soc. Org. Chem. Jpn., 23, 643 (1965).

24. T. Yamamoto, Bull. Chem. Soc. Jpn., 40, 642 (1967).

25. T. Otsu, Kogyo Kagaku Zasshi, 70, 1854 (1967).

26. M. Imoto, Kagaku, 29, 958 (1974).

27. R. L. Herbst and M. E. Jacox, J. Am. Chem. Soc., 74, 3004 (1952).

28. J. A. Landgrebe and R. H. Rynbrandt, J. Org. Chem., 31, 2585 (1966).

29. D. H. McDaniel and H. C. Brown, J. Org. Chem., 23, 420 (1958).

30. E. Kobayashi, T. Ohashi, and J. Furukawa, Makromol. Chem., 187, 2525 (1986).

31. T. Yagi, G. Morishita, and E. Kobayashi, Zairyo Gijyutsu, 7, 272 (1989).

32. E. Kobayashi, Y. Terada, T. Ohashi, and J. Furukawa, Polym. J., 23, 267 (1991). 